\title{
Body Building and Aminotransferase Elevations: A Review
}

\author{
Jaimy Villavicencio $\mathrm{Kim}^{* 1}$ and George $\mathrm{Y} . \mathrm{Wu}^{2}$ \\ ${ }^{1}$ Department of Medicine, University of Connecticut Health Center, Farmington, CT, USA; ${ }^{2}$ Division of Gastroenterology- \\ Hepatology, University of Connecticut Health Center, Farmington, CT, USA
}

\begin{abstract}
In addition to liver injury, elevation of aminotransferases can be caused by strenuous exercise and use of muscle-building and weight-loss supplements. The purpose of this review is to discuss the various mechanisms of elevation of aminotransferases related to body building. A literature review was performed on clinical trials and case reports involving exercise or supplement use and their effects on aminotransferases. Normal aminotransferase levels varied according to gender, age, body mass index, and comorbidities. Strenuous exercise and weight lifting, especially in the unaccustomed, can cause elevated aminotransferases in the absence of liver damage. Supplements such as anabolic steroids, ephedra, and LipoKinetix, amongst others, have also been associated with aminotransferase elevations. The pattern of elevation of aminotransferases is not helpful in distinguishing liver from muscle injury. Other associated muscle enzymes can be useful in making that distinction. To prevent aminotransferase elevations, subjects not accustomed to moderate-high intensity workouts, are recommended to undertake gradual increase in intensity. When causes of liver injury have been ruled out, investigation into bodybuilding, extreme exercise, and supplement use is warranted.
\end{abstract}

Citation of this article: Villavicencio Kim J, Wu GY. Body building and aminotransferase elevations: A review. J Clin Transl Hepatol 2020;8(2):161-167. doi: 10.14218/JCTH.2020.00005.

\section{Introduction}

Aminotransferases are commonly elevated with liver injury, and therefore often used as serum markers of liver pathology. Although they are often called "liver enzymes", they are not found in liver exclusively. Therefore, conditions other than liver disease should be considered as causes of elevations. Because of the increase in popularity of bodybuilding, muscle or liver injury due to strenuous exercise as well as related to use of weight loss and muscle-building supplements is increasing in frequency. The purpose of this review is to discuss the relationship between exercise and bodybuilding and elevations of aminotransferases.

Keywords: Aminotransferases; Exercise; Weight-loss supplements; Musclebuilding supplements.

Abbreviations: AAS, androgenic anabolic steroids; ALP, alkaline phosphatase; ALT, alanine aminotransferase; AST, aspartate aminotransferase; CK, creatine kinase; GGT, gamma-glutamyl transferase; LDH, lactate dehydrogenase.

Received: 20 January 2020; Revised: 23 April 2020; Accepted: 8 May 2020

*Correspondence to: Jaimy Villavicencio Kim, Department of Medicine, University of Connecticut Health Center, 263 Farmington Ave, Farmington, CT 06032, USA. Tel: +1-860-899-8739, E-mail: villavicencio@uchc.edu
Alanine and aspartate aminotransferases

Aminotransferases are enzymes that catalyze the transfer of an amino group from amino acids to oxoacids, a process known as transamination. Aspartate aminotransferase (AST; formerly known as glutamate oxaloacetate transaminase) and alanine aminotransferase (ALT; formerly termed as glutamate pyruvate transaminase) are the two aminotransferases with greatest clinical significance. Measurement of these is performed routinely for detection of hepatic disease. ${ }^{1}$

\section{Organ distribution}

In decreasing order of concentration, AST can be found in liver, heart, skeletal muscle, kidney, brain, pancreas, lungs, leukocytes, and erythrocytes. ${ }^{1}$ Up to $20 \%$ of measured AST comes from the cytosol, while $80 \%$ comes from the mitochondria. ${ }^{2}$ Cytosolic AST has a half-life of $17 \mathrm{~h}$, while mitochondrial AST has a half-life of $87 \mathrm{~h}$; although, most laboratories do not differentiate between them. ${ }^{2}$ Clearance from plasma is performed by hepatocytes, sinusoidal cells, endothelial cells, and Kupffer cells. ${ }^{3}$ Zone 3 of the hepatic acinus has higher concentrations of AST, so that damage to this zone by ischemia or toxins may result in greater levels of AST than ALT. ${ }^{1}$

Skeletal muscle and kidney contain lower concentrations of ALT than liver, and therefore, ALT elevation is more specific for liver damage. ${ }^{4}$ ALT differs from AST in that it is solely present in the cellular cytoplasm. ${ }^{1}$ Its half-life is around 47-48 h. ${ }^{1,3}$ It is also cleared by hepatocytes and nonparenchymal cells, such as Kupffer cells and endothelial cells. ${ }^{3}$

General factors affecting normal aminotransferase levels

Mera et al. ${ }^{5}$ compared, by age, females and males with normal levels of bilirubin and aminotransferases, and found significantly lower levels of AST and ALT in females compared to males in all decades of life except the $10^{\text {th }}$ and $11^{\text {th }}$ $(p<0.05)$ (Fig. 1). The median AST level was $24 \mathrm{U} / \mathrm{L}$ in females and $26 \mathrm{U} / \mathrm{L}$ in males. The median ALT was $26 \mathrm{U} / \mathrm{L}$ in females and $32 \mathrm{U} / \mathrm{L}$ in males. In the $10^{\text {th }}$ and $11^{\text {th }}$ decade, serum AST and ALT were higher in females compared to males, but this finding was not statistically significant. ${ }^{5}$ Although the patients had no known history of liver disease, other confounding factors such as comorbidities, weight and social history were not stated, leaving questions about the validity of their conclusions on gender differences.

However, in a prospective study, Bussler et al. ${ }^{6}$ also found higher levels of AST and ALT in boys compared to girls in a large sample size. In contrast with the Mera et al. study, ${ }^{5}$ 


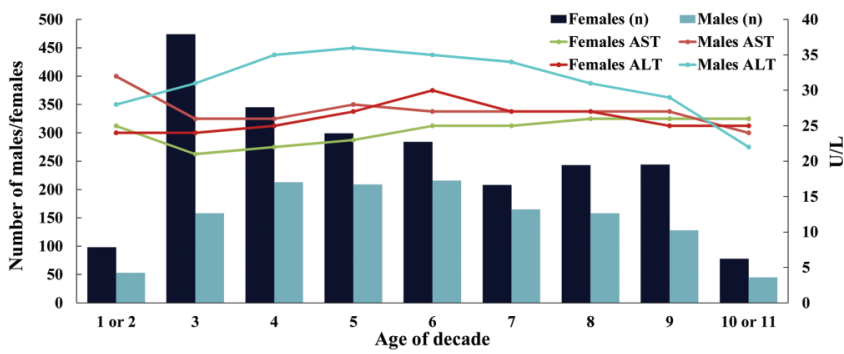

Fig. 1. A comparison of alanine aminotransferase and aspartate aminotransferase levels between females and males, stratified by decade of life.

Adapted from Mera et al. 5

these subjects were healthy, not taking any hepatotoxic medications and were neither overweight nor underweight, making these results more convincing. A peak in ALT was found corresponding to puberty in both genders. ${ }^{6}$

In a study done in healthy subjects without prior liver disease who were hospitalized for experimental reasons, ${ }^{2}$ AST and ALT levels were observed to increase $5 \%$ and $17.5 \%$ respectively above the upper limit of normal. ${ }^{7}$ This was thought to be due to restricted physical activity in combination with hospital diet. Thus, in apparently healthy patients, determining the cause of elevated aminotransferases can be a difficult diagnostic problem.

Patients who engage in bodybuilding are at risk for elevated aminotransferases due to one of several potential mechanisms, including the physical activity itself or use of supplements that induce muscle and/or liver damage.

Potential mechanisms of aminotransferase elevations related to exercise/body building

\section{Exercise-induced rhabdomyolysis}

Exercise-induced rhabdomyolysis is a common consequence of strenuous exercise. ${ }^{8,9}$ The degree of rhabdomyolysis depends on exercise experience, level of training, intensity, duration and type of workout. ${ }^{9}$ It has been found to be more common in people with less exercise experience or who were less trained. ${ }^{9}$ Significantly lower levels of creatine kinase (CK) and myoglobin have been found in highly experienced weightlifters compared to less experienced. ${ }^{9}$ Other factors that play a role are: hot environments, electrolyte imbalances, nutritional deficiencies, creatine supplements, alcohol, and gender. $^{9}$

Pal et al. ${ }^{10}$ studied sedentary teenage girls and boys with normal pre-exercise AST, ALT, and CK levels who undertook an exercise regimen. Subjects taking medications or with any underlying condition were excluded. They found that CK levels were significantly higher in boys at 24 and 48 h postexercise, with a percentage change in CK activity at $48 \mathrm{~h}$ of $84 \%$ in males and of $35 \%$ in females. ${ }^{10}$ However, there was no difference in percentage of change in AST or ALT pre- and post-exercise, at 24 or $48 \mathrm{~h}$ between genders (Table 1 ). ${ }^{10}$

Fallon et al. ${ }^{11}$ studied 7 male and 2 female subjects who had completed an ultra-marathon. They tested CK, AST, ALT pre- and post-race with follow-up tests on days 4 and 11 . Before the race, all had normal transaminases and $\mathrm{CK}$ levels. The mean value of AST, ALT and CK were above the normal range after the race on days 4 and $11 .{ }^{11}$ Although a small study, the results clearly showed that extreme exercise can elevate aminotransferases due to substantial muscle injury. Elevations in CK supported muscle injury. Co-existence of hepatocellular liver injury could not be entirely ruled out, although normal levels of alkaline phosphatase (ALP) and gamma-glutamyl transferase (GGT) made that unlikely. The enzyme levels were still elevated at day 11 , and normalization was not documented. Lactate dehydrogenase (LDH) iso-enzymes may have some value in detecting liver and muscle injury. However, although hepatocytes almost exclusively produce the $\mathrm{M}$-isoform which comprises $\mathrm{LDH}-5$, the latter is found in skeletal muscle as well as liver, which limits the value of $\mathrm{LDH}$ iso-enzymes in distinguishing liver from muscle injury. ${ }^{12}$

Apple et al. ${ }^{13}$ studied 22 male and 8 female marathon runners, testing serum markers and gastrocnemius muscle biopsies before and after the race. There was a significant increase in serum ALT levels after the race compared to normal levels prior. However, there was no elevation in ALT in gastrocnemius biopsies (which were done on three occasions), suggesting the liver was the source of serum elevations. ${ }^{13}$ The unknown sensitivities of the assays, as well as small sample sizes make this conclusion uncertain. Furthermore, other tests (i.e. ALP, CK, AST, GGT and LDH) were not

Table 1. CK, AST and ALT values before and after exercise in girls and boys ${ }^{10}$

\begin{tabular}{|c|c|c|c|c|c|c|}
\hline \multirow[b]{2}{*}{ Variable } & \multicolumn{6}{|c|}{ Mean values for $\mathrm{CK}, \mathrm{AST}, \mathrm{ALT}$} \\
\hline & $\begin{array}{l}\text { Gender } \\
\text { (Group) }\end{array}$ & $\begin{array}{l}\text { Before exercise } \\
\text { (T1) }\end{array}$ & $\begin{array}{l}\text { After exercise } \\
\text { (T2) }\end{array}$ & $\begin{array}{l}24 \mathrm{~h} \text { after exercise } \\
\text { (T3) }\end{array}$ & $\begin{array}{l}48 \mathrm{~h} \text { after exercise } \\
\text { (T4) }\end{array}$ & $\begin{array}{l}\text { \% Change (T1- } \\
\mathrm{T} 4)\end{array}$ \\
\hline \multirow[t]{2}{*}{ CK } & Boys & 139.65 & 141.18 & 253.79 & 257.4 & $84 \%$ \\
\hline & Girls & 126.98 & 128.59 & 162.47 & 168.68 & $35 \%$ \\
\hline \multirow[t]{2}{*}{ AST } & Boys & 23.95 & 25.27 & 29.2 & 30.72 & $28 \%$ \\
\hline & Girls & 18.48 & 19.5 & 23.72 & 25.51 & $38 \%$ \\
\hline \multirow[t]{2}{*}{ ALT } & Boys & 20.26 & 20.88 & 23.95 & 25.45 & $26 \%$ \\
\hline & Girls & 19.35 & 19.7 & 23.72 & 25.22 & $30 \%$ \\
\hline
\end{tabular}

Percentage of change of AST and ALT (pre- and $48 \mathrm{~h}$ post-exercise) between genders $(n=44)$.

Abbreviations: AST, aspartate aminotransferase; ALT, alanine aminotransferase; $C K$, creatine kinase.

Adapted from Pal et al. ${ }^{10}$ 
done in this study, making it difficult to differentiate between damage of liver, muscle, or both.

Unfortunately, many of the studies did not present data on follow up of participants to the point of enzyme normalization. However, Pavletic et al., Malinoski et al. and Delicata et $a .^{8,14,15}$ (amongst other authors) have presented case reports on patients with elevated ALT, AST and CK levels thought to be due to exercise. In those reports, normalization in enzyme levels was reported after discontinuation of physical activity.

\section{Pathophysiology of exertional rhabdomyolysis}

Acute high intensity exercise can induce oxidative stress and muscle damage especially in combination with other extrinsic factors, such as temperature, humidity, and medication use. ${ }^{7,16}$ Disruption of the sarcolemma can release intramuscular proteins into serum, including $\mathrm{CK}, \mathrm{LDH}$, myoglobin, aldolase, AST and ALT. ${ }^{16}$ Under resting conditions, ATPdependent ion channels keep intracellular calcium and sodium at low levels and potassium at high levels. Any insult that damages the ion channels or depletes ATP can cause an imbalance of electrolyte concentrations, increasing intracellular sodium and calcium. ${ }^{7,16}$ Likewise, with intense exercise, ATP is depleted and calcium concentration increases. These electrolyte imbalances can lead to cellular edema and activation of calcium-dependent proteases and phospholipases that ultimately result in functional degradation of cell signaling systems and decomposition of cell membrane, with release of enzymes into the extracellular space and eventually into the blood stream. ${ }^{7,16}$

Based on several case reports, it is thought that the risk of exertional rhabdomyolysis is higher with eccentric muscle training and high-intense work-outs, which may include lowweight high-repetition workouts in the unaccustomed. ${ }^{17}$ As described by Armstrong et al., ${ }^{18}$ rhabdomyolysis can occur faster with exercise in the setting of heat strokes. High temperature increases muscle membrane permeability and is, therefore, a risk factor for rhabdomyolysis. ${ }^{18}$

\section{Pattern of aminotransferase elevations in rhabdomyolysis}

A study conducted on healthy men with normal baseline laboratory tests who engaged in moderate physical activity (but not weightlifting) found elevated ALT, AST, LDH, CK and myoglobin levels at $1 \mathrm{~h}$ after heavy weightlifting. ${ }^{19}$ AST was noted to increase first, followed by ALT, with an AST/ALT ratio $>1$ at 1 week. At 10-12 days, the mean value for ALT was higher compared to AST. ${ }^{19}$ Bilirubin, GGT and ALP remained within normal limits. ${ }^{19}$ This was expected, as those enzymes are not present in muscle. Pettersson et al. ${ }^{19}$ demonstrated that weightlifting could cause muscle damage, even in subjects who were accustomed to moderate physical activity.

Weibrecht et al. ${ }^{20}$ also retrospectively studied 215 cases with rhabdomyolysis having CK greater than $1000 \mathrm{U} / \mathrm{L}$. AST was greater than $40 \mathrm{U} / \mathrm{L}$ in $93 \%$ of patients, while an abnormal ALT was only found in $75 \%$. CK and AST levels decreased in parallel, while ALT lagged. The authors excluded patients with chronically elevated aminotransferases, patients with myocardial infarction, on statin therapy, with viral hepatitis and acetaminophen toxicity. ${ }^{20}$ However, other factors such as weight, diet and medical conditions could have contributed to elevations in this retrospective study and were not ruled out.
Muscle building supplements

\section{Anabolic steroids}

Many supplements for muscle building contain androgenic anabolic steroids (AAS), whether disclosed or not. AAS are synthetic derivatives of testosterone that promote muscle growth. These can cause cholestatic liver injury, peliosis hepatis, hepatic adenoma, and hepatocellular carcinoma. ${ }^{21}$ Despite increasing efforts of the USA Food and Drug Administration, some bodybuilding supplements can still be contaminated with AAS, and the incidence of liver injury related to AAS use has been increasing. ${ }^{21}$

Anabolic steroids can cause elevation of aminotransferases up to 2-3 times the upper limit of normal. ${ }^{22}$ However, most athletes who take anabolic steroids follow an intense training regimen, so that it is often difficult to determine whether aminotransferases are elevated due to rhabdomyolysis or liver damage. With liver damage, usually GGT is elevated as well but bilirubin and CK levels are normal. ${ }^{22}$

In a prospective study, Stolz et al. ${ }^{23}$ followed 44 patients who were taking bodybuilding supplements and had elevated aminotransferases, ALP and/or bilirubin (Fig. 2). The investigators measured the medium and peak values and the percentage of increase of each laboratory test. The Drug Injury Liver Injury Network did an assessment of causality between liver injury and supplement taken based on available clinical, biochemical, radiological and histological findings at the 6-month follow-up visit. All cases were classified as 'highly likely' or 'definite', while none were deemed 'probable'. Imaging studies and additional laboratory tests (including hepatitis viral panel and autoantibodies) ruled out other liver diseases. Twenty-six patients underwent liver biopsy, of which $77 \%$ had a mixed hepatocellular and cholestatic injury and $18 \%$ had acute cholestasis. The investigators tested most of the supplements taken by the patients, but not all supplements were available, and anabolic steroids were not identified by chemical analysis among all supplements available.

From the pathology results and elimination of other causes, an association appears to have been established between certain bodybuilding supplements and cholestatic liver injury. However, this study had several limitations. It is difficult to establish a dose response to injury, and not all supplements were available for analysis. Also, other unidentified components could have contributed to liver injury. In addition, some patients were lost to follow-up, so resolution of laboratory abnormalities was not documented.

\section{Creatine supplements}

Creatine is a peptide that improves weight, strength, and muscle mass gain. It has been linked to liver damage, but the findings were not unequivocal. Whitt et al. ${ }^{24}$ described a case of acute cholestatic liver injury in a 27 year-old healthy man who was taking a combination of whey protein and creatine supplements. Liver biopsy showed marked cholestasis with duct proliferation. ${ }^{24}$ Other causes, such as exposure to solvents, recreational drug use, alcohol use, viral hepatitis and autoimmune liver disease, were ruled out. Moreover, the use of anabolic steroids was ruled out. The patient showed improvement after discontinuation of supplements, but normalization was not documented. 
A

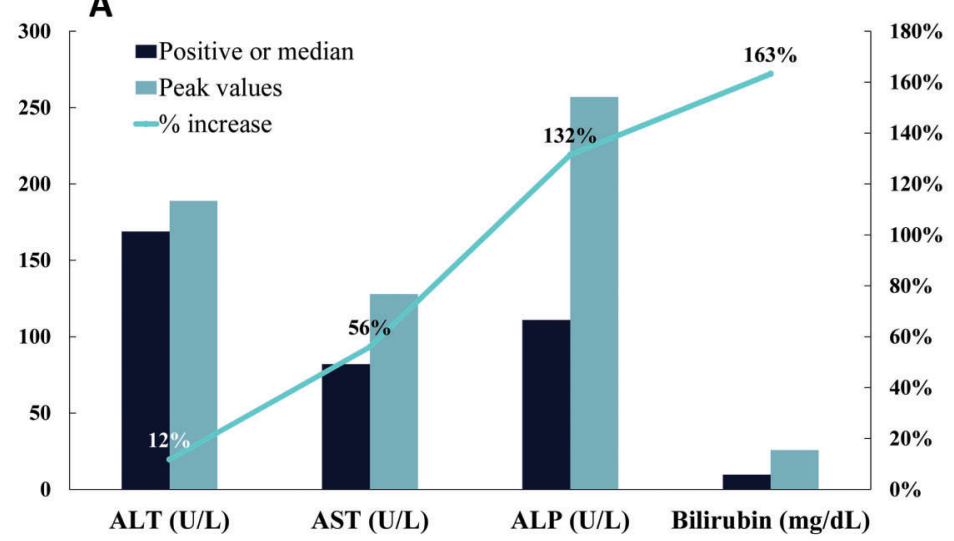

B

\section{Causality scores}

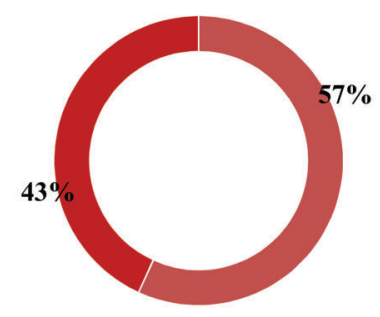

- Definite $\quad$ High likely $=$ Probable

Fig. 2. Data of 44 patients who were taking bodybuilding supplements and had elevated aminotransferases, alkaline phosphatase and/or bilirubin.

(A) Alanine aminotransferase and aspartate aminotransferase, alkaline phosphatase and bilirubin levels in patients taking bodybuilding supplements. (B) Causality scores. Adapted from Stolz et al. ${ }^{23}$

It is difficult to draw conclusions from the case reports, especially since the quantitation of creatine ingestion was generally not available. Patients usually take more than one supplement, and testing for other hepatotoxic components is usually not done. No clinical studies on the adverse effects of creatine ingested alone have been performed, making an association difficult to demonstrate. Duarte et al. and Tarnopolsky et al. ${ }^{25,26}$ found increased protein deposition and architectural changes in liver of mice supplemented with creatine. However these studies lack general applicability to a human population. Therefore, due to lack of evidence, creatine is still generally viewed as safe when taken in recommended amounts.

\section{Herbal and dietary supplements}

Stickel et al. ${ }^{27}$ reviewed cases of liver damage related to Herbalife products (Los Angeles, CA, USA). This is a brand of supplements for weight-loss and sports performance. Hepatocellular, cholestatic and mixed patterns of liver damage were described. Elinav et al. ${ }^{28}$ studied acute hepatitis of unknown cause in Israel. Twelve cases were identified with a common denominator of Herbalife product use. Infectious, autoimmune, metabolic and toxic causes of liver damage were investigated, and all patients denied illicit drug or alcohol abuse. Based on the World Health Organization criteria causality assessment, three cases were ruled as 'certain', six as 'probable' and three as 'possible'. The 'certain' cases were based on positive rechallenge, with development of a second episode of liver injury with reinitiation of supplements, and resolution with discontinuation of products. Similar results were found in a study from Switzerland. ${ }^{29}$

Despite this association, a direct causal relationship has not been drawn between Herbalife products and hepatic toxicity. All of the patients were taking more than one product, some of which could have been contaminated, possibly explaining the limited geographic distribution. Some of these patients tested positive for hepatitis B virus, antinuclear antibody, antimitochondrial antibody with biopsy-proven primary biliary cholangitis, and antismooth muscle antibody at 1:160 that became negative after recovery. Thus, there were possible confounding factors. Furthermore, accurate information regarding the ingestion of other medications was lacking.
LipoKinetix (used for weight loss; Syntrax Innovations Inc., Chaffee, MO, USA) has been associated with a hepatocellular pattern of liver injury and significant elevations of aminotransferases. ${ }^{30}$ LipoKinetix contains usnic acid, which uncouples the respiratory chain. ${ }^{30}$ These agents were withdrawn from the market after several cases of hepatitis and hepatic failure were reported to the USA Food and Drug Administration. Favreau et al. ${ }^{30}$ found seven cases of patients with hepatotoxicity after use of LipoKinetix. Three of them were taking only this supplement at time of presentation. All seven patients were healthy, with normal body mass index, taking the supplement in recommended doses, not on any other medications, and tested negative for infectious and autoimmune causes of hepatitis. Additionally, all reported cases had spontaneous recovery after discontinuation of product. ${ }^{30}$ Even though causality is challenging to prove based on case reports, these results are somewhat convincing given the common denominator. The USA Food and Drug Administration tested three of the products from different lots and ruled out contaminants, pointing towards an idiosyncratic reaction as the mechanism of injury.

Similarly, Hydroxycut products (Iovate Health Sciences, Oakville, Ontario, Canada) used for weight loss, were removed from the marked after 23 reports of acute hepatic failure, some requiring liver transplantation. ${ }^{27}$ Kaswala et al. ${ }^{31}$ reported one case of a patient using Hydroxycut, with biopsyproven acute fulminant hepatitis. Autoimmune causes were ruled out in this case, but there was no mention of whether other causes, such as viral hepatitis, were checked. ${ }^{31}$ The patient improved after stopping supplement use. ${ }^{31}$ Although it is possible the presentation was due to Hydroxycut, once again, a causal relationship was not proven.

Several other products have been associated with druginduced liver injury in case reports (Table 2). Patients usually underreport use of dietary supplements or take several supplements at once, making it challenging to pinpoint the causal agent of liver injury. ${ }^{1}$

\section{Vitamins}

DeKlotz et al. ${ }^{32}$ retrospectively examined adolescents taking isotretinoin for acne, and reported those who developed aminotransferase elevations. All of them admitted to use of 
Villavicencio Kim J. et al: Bodybuilding and aminotransferase elevations

Table 2. Bodybuilding products associated with drug-induced liver injury ${ }^{27}$

\begin{tabular}{lll}
\hline Product & Type of liver injury & Mechanism \\
\hline $\begin{array}{l}\text { LipoKinetix } \\
\text { steroids }\end{array}$ & $\begin{array}{l}\text { Acute hepatitis } \\
\text { Cholestasis, benign/malignant tumors }\end{array}$ & $\begin{array}{l}\text { Possibly uncoupling of the respiratory chain } \\
\text { Dysfunction of biliary transport }\end{array}$ \\
$\begin{array}{l}\text { Noni juice } \\
\text { Senna }\end{array}$ & $\begin{array}{l}\text { Acute hepatitis, liver failure } \\
\text { Acute hepatitis, granulomatous hepatitis, } \\
\text { cirrhosis }\end{array}$ & $\begin{array}{l}\text { Unknown } \\
\text { Possibly drug idiosyncrasy or uncoupling of the respiratory } \\
\text { chain }\end{array}$ \\
$\begin{array}{l}\text { Green tea } \\
\text { Ephedra }\end{array}$ & $\begin{array}{l}\text { Acute hepatitis } \\
\text { Acute hepatitis, liver failure }\end{array}$ & $\begin{array}{l}\text { Possibly oxidative stress from epigallocatechin gallate } \\
\text { Unknown }\end{array}$ \\
\hline
\end{tabular}

Adapted from Stickel et al. $^{27}$

herbal, protein or creatine supplementation, and some of them had initiated vitamin A therapy at the time. There were many confounding factors in this study, so it is difficult to determine if aminotransferase elevations were due to liver damage or muscle damage. There was no specific pattern in elevation of AST, ALT or supporting laboratory testing (such as CK). Nevertheless, because vitamin A alone is known to cause hepatic injury, ${ }^{33}$ it is certainly possible that in combination with an underlying medical condition, alcohol use, medications or genetic predisposition, its use resulted in an increased risk of liver damage.

Potential causes of rhabdomyolysis with exercise/ body building

\section{Ischemia}

Conditions with generalized ischemia and hypoxemia can cause insufficient ATP production and sarcolemma dysfunction. ${ }^{34}$ Causes include but are not limited to: shock, arterial thrombosis, air emboli, sickle cell disease, and status asthmaticus. ${ }^{34}$ Compartment syndrome can be a cause or complication of rhabdomyolysis due to impaired blood flow. ${ }^{34}$ Prolonged immobilization causes tissue compression and muscle ischemia as well. ${ }^{34}$ Severe dehydration, especially in the setting of heat stroke and exercise can also cause rhabdomyolysis. ${ }^{34}$

\section{Muscle building supplements}

Creatine supplements can result in rapid weight gain due to intracellular and extracellular fluid retention. ${ }^{35}$ Robinson et al. ${ }^{35}$ speculated that increased intracellular water retention caused greater skeletal muscle compartment pressures, which increased risk of cellular wall breakdown. The first association of creatine supplementation and rhabdomyolysis was made in 1997 after three wrestlers died while on creatine supplements. ${ }^{35}$ However, they were also using ephedra supplements for weight loss. Several studies have reported no effect of high-dose short-term or low-dose long-term creatine use in physically unstressed subjects or power athletes on high-dose creatine. ${ }^{35-37}$

Despite these studies, there are few cases reported of rhabdomyolysis in the setting of creatine supplement use. The majority were involved in extreme exercise regimens. Some subjects also ingested ephedrine or herbal supplements. In the setting of extreme, unaccustomed exercise and usage of other supplements, it is difficult to prove creatine as the culprit.

\section{Weight loss supplements}

The sympathomimetic amine ephedra was banned in 2004 after numerous reports of cardiovascular and neurologic adverse effects. ${ }^{38}$ Synephrine became a popular alternative, due to its structural similarity to ephedrine. It is thought to increase the risk of rhabdomyolysis through vasoconstriction and vasospasm, causing ischemia, direct toxicity, and impairment of calcium homeostasis or myocyte thermoregulatory function. ${ }^{38}$

Burke et al. ${ }^{38}$ reported a case of a male subject who engaged in vigorous exercise and ingested a weight loss supplement containing synephrine and caffeine. He developed rhabdomyolysis with elevated CK and aminotransferases on two different occasions. During his first hospitalization, he was not queried regarding use of supplements and continued to use Lipo 6 twice daily after discharge until his second presentation. Even though he had several predisposing risk factors, such as sickle cell trait, a previous episode of rhabdomyolysis, and exercise in warm climate, it is important to note that prior to use of supplements, there was no rhabdomyolysis in spite of the use of same exercise regimen. Although this may represent direct muscle injury by synephrine, the association has not been proven.

A 40-year-old man was reported to have developed rhabdomyolysis after taking Garcinia cambogia. ${ }^{39}$ Also known as Malabar tamarind, this tropical fruit is a popular weight loss inducer. The patient denied use of prescription medications, rigorous exercising, other supplements or dehydration. This is the only case report where a single-ingredient supplement with Garcinia cambogia was associated with rhabdomyolysis. However, although suggestive, there is insufficient evidence to establish a causal relationship. Other factors might have predisposed the patient to muscle damage.

\section{Preventative measures}

Hill et al. ${ }^{40}$ found that the strongest risk factors for rhabdomyolysis in army soldiers were prior heat stroke, black race and length of stay of less than 90 days. Although confounding factors such as hydration, temperature and humidity (which are known to increase risk for rhabdomyolysis) $)^{7,40}$ were not considered, it seemed new recruits had double the likelihood 
compared to soldiers who were there for more than 90 days. ${ }^{40}$ Those with length of stay greater than 1 year had an odds ratio of developing rhabdomyolysis below 1.00 .40 This suggests that subjects who are unaccustomed to exercise have higher risk of muscle damage. Similarly, Oh et al. ${ }^{41}$ described athletes who developed rhabdomyolysis 1 day after starting an intense exercise regimen in a football camp. Questionnaires were handed to athletes and those who were voted for as 'hardest working' had a relative risk of 2.1 compared to the group that did less effort. ${ }^{41}$ The athletes denied drugs or medications and their aerobic and resistance exercises months prior were similar. Given that these athletes performed the same exercises under the same environmental conditions, the study suggests that intense training for firsttimers or after a training hiatus could potentially increase the risk for rhabdomyolysis. It is reasonable to recommend a slow buildup to intensity of exercise desired in subjects who are not used to moderate-high intensity workouts. Since temperature is a risk factor, avoiding hot climates and wearing adequate clothing that aid heat dissipation might be protective.

Measures such as warm-ups, sufficient water intake and ingestion of protein in combination with carbohydrates can help prevent rhabdomyolysis. ${ }^{7,40,42,43}$ Baty et al. ${ }^{44}$ gave carbohydrate-protein supplements to a group of athletes and compared them to another group which received a placebo (electrolytes and artificial sweetener). They measured performance and muscle damage and found CK and myoglobin levels were significantly higher in the placebo group $24 \mathrm{~h}$ after exercise. ${ }^{44} \mathrm{~A}$ health history was obtained from each participant, those taking enhancing supplements were excluded and they started performing the same training sessions weeks prior to the start of the study. ${ }^{44}$ Because they were all under the same environment, and even had the same diet prior to start of exercise, these results seem convincing.

The type of exercise that should be done in order to prevent rhabdomyolysis is unclear, although it is known that eccentric contraction may cause more rhabdomyolysis than concentric contraction. ${ }^{42,45}$ Stretching and warm-ups are also generally thought to decrease the incidence and likelihood of muscle injuries due to increase in flexibility and range of motion. ${ }^{46}$ Small et al. ${ }^{46}$ performed a systematic review to assess efficacy of static stretching as part of warm-up to prevent exertional rhabdomyolysis. They found that all randomized clinical trials and two out of three controlled clinical trials did not find a significant difference in all-injuries risk between control and intervention group. However, the hazard ratios from five of the seven studies would indicate that stretching reduces risk of muscular strains and ligament sprains. It might be reasonable to perform these activities prior to a more intense work-out, but there is no convincing evidence that it would prevent rhabdomyolysis.

\section{Conclusions}

Aminotransferases are commonly associated with liver disease, but can also be elevated secondary to exercise and supplement use in athletes and non-athletes. A history of new or recently intensified exercise regimen should prompt a search for muscle injury. The coexistence of elevated cholestatic serum markers, such as ALP, GGT, and 5'-nucleotidease, can be useful in diagnosing liver damage. Elevated levels of markers of muscle injury, including CK, can be helpful in diagnosing muscle injury. The pattern of elevation of aminotransferases is not valuable in distinguishing muscle from liver injury as it can vary depending on the number of days after injury when testing is done. Therefore, when causes of liver injury have been ruled out, investigation into muscle injury associated with bodybuilding, and supplement use is warranted.

\section{Acknowledgements}

The support of the Herman Lopata Chair in Hepatitis is gratefully acknowledged.

\section{Funding}

None to declare.

\section{Conflict of interest}

The authors have no conflict of interests related to this publication.

\section{Author contributions}

Wrote the manuscript and prepared figures (JVK), proposed the idea for the review and revised the manuscript with critical revisions (GYW).

\section{References}

[1] Vroon DH, Israili Z. Aminotransferases. In: Walker HK, Hall WD, Hurst JW, editors. Clinical methods: The history, physical, and laboratory examinations. $3^{\text {rd }}$ edition. Boston: Butterworths; 1990.

[2] Giannini EG, Testa R, Savarino V. Liver enzyme alteration: a guide for clinicians. CMAJ 2005;172:367-379. doi: 10.1503/cmaj.1040752.

[3] Radi ZA, Koza-Taylor PH, Bell RR, Obert LA, Runnels HA, Beebe JS, et al. Increased serum enzyme levels associated with kupfer cell reduction with no signs of hepatic or skeletal muscle injury. Am J Pathol 2011;179:240247. doi: 10.1016/j.ajpath.2011.03.029.

[4] Giboney PT. Mildly elevated liver transaminase levels in the asymptomatic patient. Am Fam Physician 2005;71:1105-1110.

[5] Mera JR, Dickson B, Feldman M. Influence of gender on the ratio of serum aspartate aminotransferase (AST) to alanine aminotransferase (ALT) in patients with and without hyperbilirubinemia. Dig Dis Sci 2008;53:799802. doi: 10.1007/s10620-007-9924-z.

[6] Bussler S, Vogel M, Pietzner D, Harms K, Buzek T, Penke M, et al. New pediatric percentiles of liver enzyme serum levels (alanine aminotransferase, aspartate aminotransferase, $\gamma$-glutamyltransferase): Effects of age, sex, body mass index, and pubertal stage. Hepatology 2018;68:1319-1330. doi: 10.1002/hep.29542.

[7] Narjes H, Nehmiz G. Effect of hospitalisation on liver enzymes in healthy subjects. Eur J Clin Pharmacol 2000;56:329-333. doi: 10 . 1007/s002280000142.

[8] Pavletic AJ, Pao M. Exercise-induced elevation of liver enzymes in a healthy female research volunteer. Psychosomatics 2015;56:604-606. doi: 10 . 1016/j.psym.2015.03.002.

[9] Kim J, Lee J, Kim S, Ryu HY, Cha KS, Sung DJ. Exercise-induced rhabdomyolysis mechanisms and prevention: A literature review. J Sport Health Sci 2016;5:324-333. doi: 10.1016/j.jshs.2015.01.012.

[10] Pal S, Chaki B, Chattopadhyay S, Bandyopadhyay A. High-intensity exercise induced oxidative stress and skeletal muscle damage in postpubertal boys and girls: A comparative study. J Strength Cond Res 2018;32:1045-1052. doi: $10.1519 /$ JSC.0000000000002167.

[11] Fallon KE, Sivyer G, Sivyer K, Dare A. The biochemistry of runners in a 1600 km ultramarathon. Br J Sports Med 1999;33:264-269. doi: 10.1136/bjsm. 33.4.264.

[12] Puri BK, Kingston MC, Monro JA. Fructose-associated hepatotoxicity indexed by the lactate dehydrogenase isoenzyme LDH-5. Med Hypotheses 2019; 124:40-41. doi: 10.1016/j.mehy.2019.02.019.

[13] Apple FS, Rogers MA. Serum and muscle alanine aminotransferase activities in marathon runners. JAMA 1984;252:626-627. doi: 10.1001/jama.1984. 03350050018012. 
[14] Malinoski FJ. Strenuous exercise simulating hepatic injury during vaccine trials. Vaccine 1992;10:39-42. doi: 10.1016/0264-410x(92)90417-i.

[15] Delicata NP, Delicata J, Delicata LA. Strenuous exercise-An unusual cause of deranged liver enzymes. Case Reports in Clinical Medicine 2018;7:177-181. doi: $10.4236 / \mathrm{crcm} .2018 .73016$

[16] Torres PA, Helmstetter JA, Kaye AM, Kaye AD. Rhabdomyolysis: pathogenesis, diagnosis, and treatment. Ochsner J 2015;15:58-69.

[17] Tran M, Hayden N, Garcia B, Tucci V. Low-intensity repetitive exercise induced rhabdomyolysis. Case Rep Emerg Med 2015;2015:281540. doi: $10.1155 / 2015 / 281540$.

[18] Armstrong LE, Casa DJ, Millard-Stafford M, Moran DS, Pyne SW, Roberts Wo. American College of Sports Medicine position stand. Exertional heat illness during training and competition. Med Sci Sports Exerc 2007;39:556-572. doi: 10.1249/MSS.0b013e31802fa199.

[19] Pettersson J, Hindorf U, Persson P, Bengtsson T, Malmqvist U, Werkström $V_{,}$ et al. Muscular exercise can cause highly pathological liver function tests in healthy men. Br J Clin Pharmacol 2008;65:253-259. doi: 10.1111/j.13652125.2007.03001.x.

[20] Weibrecht K, Dayno M, Darling C, Bird SB. Liver aminotransferases are elevated with rhabdomyolysis in the absence of significant liver injury. J Med Toxicol 2010;6:294-300. doi: 10.1007/s13181-010-0075-9

[21] Bond P, Llewellyn W, Van Mol P. Anabolic androgenic steroid-induced hepatotoxicity. Med Hypotheses 2016;93:150-153. doi: 10.1016/j.mehy.2016. 06.004.

[22] Niedfeldt MW. Anabolic steroid effect on the liver. Curr Sports Med Rep. 2018; 17:97-102. doi: 10.1249/JSR.0000000000000467.

[23] Stolz A, Navarro V, Hayashi PH, Fontana RJ, Barnhart HX, Gu J, et al. Severe and protracted cholestasis in 44 young men taking bodybuilding supplements: assessment of genetic, clinical and chemical risk factors. Aliment Pharmacol Ther 2019;49:1195-1204. doi: 10.1111/apt.15211.

[24] Whitt KN, Ward SC, Deniz K, Liu L, Odin JA, Qin L. Cholestatic liver injury associated with whey protein and creatine supplements. Semin Liver Dis 2008;28:226-231. doi: 10.1055/s-2008-1073122.

[25] Duarte JA, Neuparth MJ, Soares JMC, Appell HJ. Oral creatine supplementation in mice induces hepatic protein overload. Revista Portuguesa de Ciências do Desporto $2001 ; 1: 40-43$. doi: 10.5628/rpcd.01.03.40.

[26] Tarnopolsky MA, Bourgeois JM, Snow R, Keys S, Roy BD, Kwiecien JM, et al. Histological assessment of intermediate- and long-term creatine monohydrate supplementation in mice and rats. Am J Physiol Regul Integr Comp Physiol 2003;285:R762-R769. doi: 10.1152/ajpregu.00270.2003.

[27] Stickel F, Kessebohm K, Weimann R, Seitz HK. Review of liver injury associated with dietary supplements. Liver Int 2011;31:595-605. doi: $10.1111 / \mathrm{j}$. 1478-3231.2010.02439.x.

[28] Elinav E, Pinsker G, Safadi R, Pappo O, Bromberg M, Anis E, et al. Association between consumption of Herbalife nutritional supplements and acute hepatotoxicity. J Hepatol 2007;47:514-520. doi: 10.1016/j.jhep.2007.06.016.

[29] Schoepfer AM, Engel A, Fattinger K, Marbet UA, Criblez D, Reichen J, et al. Herbal does not mean innocuous: ten cases of severe hepatotoxicity associated with dietary supplements from Herbalife products. J Hepatol 2007;47: 521-526. doi: 10.1016/j.jhep.2007.06.014.

[30] Favreau JT, Ryu ML, Braunstein G, Orshansky G, Park SS, Coody GL, et al. Severe hepatotoxicity associated with the dietary supplement LipoKinetix.
Ann Intern Med 2002;136:590-595. doi: 10.7326/0003-4819-136-8200204160-00008.

[31] Kaswala D, Shah S, Patel N, Raisoni S, Swaminathan S. Hydroxycut-induced liver toxicity. Ann Med Health Sci Res 2014;4:143-145. doi: 10.4103/21419248.126627.

[32] DeKlotz CMC, Roby KD, Friedlander SF. Dietary supplements, isotretinoin, and liver toxicity in adolescents: A retrospective case series. Pediatrics 2017;140:e20152940. doi: 10.1542/peds.2015-2940.

[33] Geubel AP, De Galocsy C, Alves N, Rahier J, Dive C. Liver damage caused by therapeutic vitamin A administration: estimate of dose-related toxicity in 41 cases. Gastroenterology 1991;100:1701-1709. doi: 10.1016/0016-5085 (91)90672-8.

[34] Efstratiadis G, Voulgaridou A, Nikiforou D, Kyventidis A, Kourkouni E, Vergoulas G. Rhabdomyolysis updated. Hippokratia 2007;11:129-137.

[35] Robinson TM, Sewell DA, Casey A, Steenge G, Greenhaff PL. Dietary creatine supplementation does not affect some haematological indices, or indices of muscle damage and hepatic and renal function. Br J Sports Med 2000;34: 284-288. doi: 10.1136 /bjsm.34.4.284.

[36] Rawson ES, Clarkson PM, Tarnopolsky MA. Perspectives on exertional rhabdomyolysis. Sports Med 2017;47:33-49. doi: 10.1007/s40279-017-0689-z.

[37] Mihic S, MacDonald JR, McKenzie S, Tarnopolsky MA. Acute creatine loading increases fat-free mass, but does not affect blood pressure, plasma creatinine, or CK activity in men and women. Med Sci Sports Exerc 2000;32:291296. doi: 10.1097/00005768-200002000-00007.

[38] Burke J, Seda G, Allen D, Knee TS. A case of severe exercise-induced rhabdomyolysis associated with a weight-loss dietary supplement. Mil Med 2007; 172:656-658. doi: 10.7205/milmed.172.6.656.

[39] Hines EQ, Thomas ED, Melville LD, Su MK. Severe rhabdomyolysis associated with Garcinia cambogia. Clinical Toxicology 2015;53:746-747.

[40] Hill OT, Scofield DE, Usedom J, Bulathsinhala L, McKinnon C, Kwon P, et al. Risk factors for rhabdomyolysis in the U.S. Army. Mil Med 2017;182:e1836e1841. doi: 10.7205/MILMED-D-16-00076.

[41] Oh JY, Laidler M, Fiala SC, Hedberg K. Acute exertional rhabdomyolysis and triceps compartment syndrome during a high school football cAMP. Sports Health 2012;4:57-62. doi: 10.1177/1941738111413874.

[42] Rider BC, Coughlin AM, Carlson C, Hew-Butler T. Exertional (exerciseinduced) rhabdomyolysis. ACSM's Health \& Fitness Journal 2019;23:1620. doi: 10.1249/FIT.0000000000000478.

[43] Hannah-Shmouni F, McLeod K, Sirrs S. Recurrent exercise-induced rhabdomyolysis. CMAJ 2012;184:426-430. doi: 10.1503/cmaj.110518.

[44] Baty JJ, Hwang H, Ding Z, Bernard JR, Wang B, Kwon B, et al. The effect of a carbohydrate and protein supplement on resistance exercise performance, hormonal response, and muscle damage. J Strength Cond Res 2007;21: 321-329. doi: 10.1519/R-21706.1.

[45] Fridén J, Sfakianos PN, Hargens AR. Muscle soreness and intramuscular fluid pressure: comparison between eccentric and concentric load. J Appl Physiol (1985) 1986;61:2175-2179. doi: 10.1152/jappl.1986.61.6.2175.

[46] Small K, Mc Naughton L, Matthews M. A systematic review into the efficacy of static stretching as part of a warm-up for the prevention of exercise-related injury. Res Sports Med 2008;16:213-231. doi: 10. $1080 / 15438620802310784$. 